\title{
Optimizing quantitative myocardial perfusion by positron emission tomography for guiding CAD management
}

\author{
K. Lance Gould, $M D^{a}$ \\ ${ }^{a}$ Weatherhead PET Center for Preventing and Reversing Atherosclerosis, McGovern Medical \\ School, University of Texas Health Science Center at Houston, Houston, TX
}

Received Aug 27, 2016; accepted Aug 29, 2016

doi:10.1007/s12350-016-0666-1

\section{See related article, pp. $1941-1949$}

\section{WHAT DOES THE REPORT TELL US?}

In the current issue, Lassen et al. ${ }^{1}$ studied the effect of respiratory gating on myocardial stress perfusion imaging using positron emission tomography with $\mathrm{Rb}-82$ in 48 patients randomized to adenosine or dipyridamole stress. The dipyridamole group consisted of non-smoking women without a family history of cardiovascular disease and with a mean age of 64 years. The adenosine group consisted primarily of men $(86 \%)$ at a mean age of 57 years of whom $39 \%$ were current smokers and $48 \%$ had a family history of cardiovascular disease.

The time-based respiratory gating method divides the respiratory signal into 8 time-equal bins determined by either the inspiratory or the expiratory peak. The timebased binning is based on the assumption of a steady, nonchanging respiratory rate and depth throughout the PET acquisition often characterizing resting conditions, but not during stress image acquisition since pharmacologic stress agents differentially alter the respiratory pattern.

\footnotetext{
Funding KLG received internal funding from the Weatherhead PET Center for Preventing and Reversing Atherosclerosis; and is the 510(k) applicant for CFR Quant (K113754) and HeartSee (K143664), software packages for cardiac positron emission tomography image processing and analysis, including absolute flow quantification.

Reprint requests: K. Lance Gould, MD, Weatherhead PET Center for Preventing and Reversing Atherosclerosis, McGovern Medical School, University of Texas Health Science Center at Houston, 6431 Fannin St., Room MSB 4.256, Houston, TX, 77030, k.lance.gould@uth.tmc.edu

J Nucl Cardiol 2017;24:1950-4.

$1071-3581 / \$ 34.00$

Copyright (C) 2016 American Society of Nuclear Cardiology.
}

\section{THE CONCLUSIONS}

The authors conclude that patients undergoing adenosine stress showed a decrease in measured respiratory rate from initial to later scan phase measurements $(12.4 \pm 5.7$ vs $5.6 \pm 4.7$ per minute, $p<0.001)$ and had a lower frequency of successful respiratory gating compared to dipyridamole ( $47 \%$ vs $71 \%, p=0.12$ ). As a result of the varying respiratory pattern with adenosine, the adenosine imaging quality was inferior to dipyridamole, although dipyridamole respiratory gating still remained unsatisfactory in $29 \%$ of dipyridamole cases. If respiratory gating is considered for use in cardiac PET, the authors recommend dipyridamole stress due to its more uniform respiration pattern than adenosine with resulting higher frequency of successful respiratory gating that, however, still remains unsatisfactory in $29 \%$ of dipyridamole cases.

\section{IMPLICATIONS}

This carefully done study is practically important as an essentially "negative" by showing definitively that respiratory gating of PET perfusion images is not useful, and may be worse than no respiratory gating due to physiologic varying respiratory rates particularly during adenosine stress but also to a significant extent with dipyridamole stress. Thus, the most important observation is physiology, the variable respiratory patterns after adenosine with $53 \%$ failed respiratory gating and $29 \%$ failure for dipyridamole. These observations preclude useful respiratory gating for PET myocardial perfusion for physiologic reasons unrelated to any systematic PET data that are not reported.

\section{IS WHAT IS MISSING IMPORTANT?}

In addition to the systematic physiologic data, the single figure of PET images with successful respiratory 
gating shows a substantial difference between the septal and lateral walls not present on images with no respiratory gating. This regional discordance of relative myocardial uptake of $\mathrm{Rb}-82$ on one example raises the question of whether even "successful" respiratory gating causes artifacts as reported for misregistration of emission and CT transmission data for attenuation correction. While the physiologic observations on respiratory variation during vasodilator stress are definitive, its negative finding might be even stonger by quantifying the differences in relative myocardial uptake, in absolute myocardial perfusion or CFR with and without respiratory gating since the study was done with PET imaging. On the other hand, the physiologic observation is so definitive that quantitative PET data might be superfluous.

While not likely to change the conclusion, the patient selection for adenosine versus dipyridamole stress is a curious bias at odds with the meticulous, objective methodology and data on respiratory variability. No explanation is provided for using dipyridamole in women and primarily adenosine in men. This striking difference is not likely random but appears to reflect some guidelines or practice pattern for the author's PET protocols that are not reported. However, again the definitive physiologic data make this odd selection of subjects of little import.

\section{OTHER OBSERVATIONS ON METHODOLOGY OF THE STUDY}

Other methodology variations needing mention are the vasodilator stress protocols. Adenosine is infused for 6 min but with imaging starting at $2.5 \mathrm{~min}$ rather than the $3 \mathrm{~min}$ that is the official protocol recommended by the ASNC/ACC/AHA procedure guidelines. $^{2}$ This earlier $30 \mathrm{~s}$ start of imaging may be due to the time required for infusion of Rb-82. However, the methods statement is that Imaging was started at $2.5 \mathrm{~min}$, thereby implying that the $\mathrm{Rb}-82$ infusion would have been started at $2 \mathrm{~min}$ in order to image myocardial uptake at $2.5 \mathrm{~min}$, a detail that is not made clear. This timing might alter the conclusions, if the last $3 \mathrm{~min}$ of a $6 \mathrm{~min}$ adenosine infusion during the standard time of radionuclide uptake had less respiratory variation than the first $3 \mathrm{~min}$.

In a parallel variation, the dipyridamole protocol used the standard dose of $0.56 \mathrm{mg} / \mathrm{k}$, but was given as a "slow bolus" with imaging at $6 \mathrm{~min}$ in contrast to the standard infusion over 4 min with imaging at $8 \mathrm{~min}^{2}$ However, these small details are not likely to change the conclusions.

\section{CLINICAL MYOCARDIAL PERFUSION PET FOR GUIDING MANAGEMENT AND PROCEDURES IN CAD}

The authors definitively address a specific, highly focused question on the usefulness of cardiac PETrespiratory gating. While accepting that focus as good scientific design for getting a definitive answer in this paper, that highly specific focus leaves open the broader issue of optimal PET methodology for quantifying stress flow and Coronary Flow Reserve as definitive guides to managing coronary artery disease (CAD).

Physiologic severity of coronary artery stenosis by invasive pressure-derived Fractional Flow Reserve (FFR) is the current "gold standard" for guiding PCI. ${ }^{3,4}$ However, FFR is only a relative flow reserve measurement that was originally validated by comparison to absolute stress perfusion and Coronary Flow Reserve by PET. ${ }^{5}$ Accordingly, in the spirit of this meticulous, focused, critical study on respiratory-gated cardiac PET, this editorial provides a perspective on six broad principles for optimal technical quantitative PET perfusion imaging as the technical basis for optimally guiding management and procedures in CAD.

\section{ATTENTUATION-EMISSION CO-REGISTRATION}

The first common error in myocardial perfusion PET is emission-attenuation misregistration due to cardiac and respiratory motion that cause significant quantified artifactual perfusion defects in $20 \%$ or more of PET-CT scans. ${ }^{6-9}$ In addition to respiratory variation, cardiac motion includes systolic-diastolic wall thickening that alters partial volume loss and translation of the heart recoiling downward and medially toward the mediastinum during systole. ${ }^{6-9}$ Consequently, ECG-gated systolic images have significantly higher activity recovery than diastolic images, thereby allowing better partial volume correction for $\mathrm{PET}^{10}$ and a greater than $50 \%$ reduction of false positives on SPECT scans. ${ }^{11}$ ECGgated perfusion images also provide accurate measures of ejection fraction. However, routinely checking and correcting attenuation-emission co-registration on every PET scan is not routinely done in many PET facilities.

\section{ARTERIAL ACTIVITY INPUT FUNCTION FOR QUANTITATIVE PERFUSION}

Second, the selection of the ROI for arterial input to determine absolute perfusion in $\mathrm{cc} / \mathrm{min} / \mathrm{gm}$ and Coronary Flow Reserve is perhaps the most critical and most neglected of all the technical elements of quantitative myocardial perfusion ${ }^{12,13}$ for several reasons. No one 
fixed ROI is optimal for all patients. Arterial input ROIs located on late myocardial images back projected onto early first-pass images are commonly not in the left atrium during significant portions of the heart cycle due to heart and respiratory motion. Spillover activity from adjacent subclavian veins and pulmonary artery branches frequently contaminates ROIs back projected from late myocardial images due to imprecise location over the left atrium.

Multiple, short, brief (15 s) first-pass arterial input images are so noisy with poor count density that ROIs cannot be optimally directly located over left atrial or aortic activity uncontaminated by adjacent subclavian veins or pulmonary artery branches. Locating ROIs over the LV gives consistently erroneous arterial input values due to LV translation into and out of the fixed ROI during the cardiac cycle and due to spillover from myocardial activity during late arterial activity acquisition. ${ }^{13}$

Variable suboptimal ROI selection causes great variability of quantitative perfusion. Therefore, we use semi-automated software to locate rapidly 5 to 6 ROIs directly on high-quality 2-min first-pass images in the ascending and descending aorta, the high, mid, and low left atrium, and in the pulmonary artery, and right atrium to bracket the maximum activity that correct ROIs must remain below. The highest activity of these alternative ROIs without contamination by readily identified subclavian or pulmonary artery branches provide the optimal ROI and arterial input for each individual patient with resulting reproducible, clinically reliable measurements of perfusion and CFR. ${ }^{13}$

\section{MAXIMUM PHARMACOLOGIC STRESS IS ESSENTIAL FOR GUIDING CAD INTERVENTIONS}

Third, maximum stress is essential since submaximal stress causes erroneous low stress flow misleadingly suggesting diffuse or focal low stress flow due to diffuse disease or focal stenosis. Such "poor stress" images are essentially false positives since repeat PET with maximal stress shows high coronary flow capacity. Measurable blood caffeine is the commonest cause in approximately 4000 quantitative PET studies in the Weatherhead PET Center such that we measure blood caffeine in all patients and repeat the study if critical for a clinical decision based on quantitative perfusion. The standard regadenoson protocol with radionuclide injection at 10 to $20 \mathrm{~s}$ after a $10 \mathrm{~s}$ infusion causes stress perfusion that is $20 \%$ lower than maximum stress perfusion by the 4-8 min dipyridamole or the 3-6 min adenosine protocols. ${ }^{14}$ Regadenoson, therefore, fails to demonstrate true severity on relative images and erroneously suggests worse absolute stress flow capacity and more severe disease than seen with maximum stress.

\section{D VERSUS 3D PET-CT SCANNERS}

Fourth, widespread 2D PET-CT scanner adequately acquire the first-pass arterial input of 35 to $40 \mathrm{mCi}$ of $\mathrm{Rb}-82$ for absolute perfusion in $\mathrm{cc} / \mathrm{min} / \mathrm{gm}$ and CFR. However, all current 3D PET-CT scanners saturate with this first-pass arterial concentration of RB-82 such that only 20 to $25 \mathrm{mCI}$ can be given with resulting count poor images and suboptimal regional stress flow and CFR in our experience. ${ }^{15,16}$ Consistent with our observation, centers using 3D PET-CT scanners usually report global stress perfusion and CFR, not regional quantitative perfusion, but the topic remains debated. Likely still newer generation of 3D scanners not currently on the market may improve this first-pass capacity for Rb-82, while maintaining high-quality late myocardial images adequate for regional quantitative perfusion. On the other hand, the dose of $\mathrm{N}-13$ ammonia is low enough at 10 to $20 \mathrm{mCi}$ that virtually all current 3D scanners measure the first-pass arterial input accurately without saturating the scanner.

\section{FLOW MODELS FOR QUANTITATIVE PERFUSION}

Fifth, flow models for calculating absolute myocardial perfusion are specific for each radionuclide and are well validated for Rb-82, N-13 ammonia, and oxygen15. The argument that greater extraction fraction by the myocardium yields the "best" quantitative perfusion is nonsense because the flow-dependent extraction or trapping of each of these radionuclides is accounted for in the flow model. Each of these radionuclides has been proven to measure myocardial perfusion accurately, despite their widely different extraction or myocardial trapping characteristics.

The advantages of the "simple" or "retention" model of Yoshida et al. ${ }^{17}$ are high-quality 2-min firstpass images that provide for direct, robust, optimal individualized arterial ROI and arterial input function for individual patients compared to multi-compartmental models ${ }^{16,17}$ with resulting "higher sensitivity for detection and localization of abnormal flow and myocardial perfusion reserve... without the computational complexity and sensitivity to noise - of the multi-compartmental model.",16

\section{CINE CT VERSUS HELICAL CT FOR ATTENUATION CORRECTION}

Sixth, a single, seconds-long helical CT image for attenuation correction may not match the average attenuation structures altering the emission image acquired over 5 min for Rb-82 due to respiratory and cardiac 


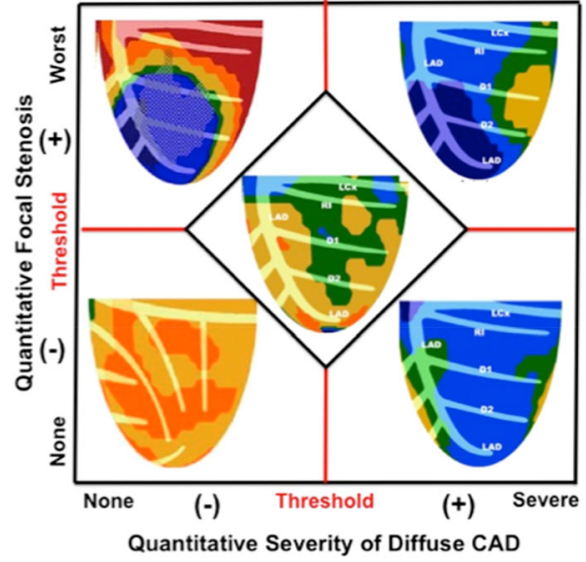

Normal flow Minimally reduced, No ischemia Mildly reduced, No ischemia

Moderately reduced, No ischemia Severely reduced, Ischemia Myocardial Steal, Ischemia

Figure 1. Patient selection for severity-stratified randomized trial using quantitative flow capacity maps based on ischemic flow thresholds for focal and diffuse disease: severe stenosis with no diffuse disease (upper left), severe stenosis with severe diffuse disease (upper right), severe diffuse disease with no segmental stenosis (lower right) and mild to moderate diffuse disease without significant stenosis (lower left) and moderate diffuse disease with intermediate stenosis neither at low ischemic flow levels (central diamond). Color-coded categories of flow capacity are based on absolute myocardial perfusion capacity for rest flow, stress flow, and coronary flow reserve (CFR) (reproduced with permission from reference 20).

motions. Even shifting seconds-long helical image data to superimpose them may not be satisfactory in some cases due to the helical CT scan capturing only on moment within the cardiac and respiratory cycles. However, cine CT or its equivalent captures the average attenuation corrections over several respiratory and cardiac cycles, thereby matching the emission images. ${ }^{7-9}$

\section{STANDARDS FOR QUANTITATIVE PET TO GUIDE CORONARY INTERVENTIONS}

While many PET centers do not use the technical protocols outlined here, this editorial honors and reflects the careful, objective, focused study on respiratory gating in this issue; the kind of critical self-examination of one's own methods for errors; and suboptimal performance that leads to improvement, proof of reproducibility, and relevance to documented ischemia by angina and ST depression during vasodilator stress. ${ }^{18,19}$ Figure 1 illustrates quantitative PET that defines physiologic severity of focal and diffuse disease based on documented ischemic thresholds of severity. ${ }^{20}$ The color-coded severity predicts progressive risk of major adverse coronary events (MACE) including PCI or coronary bypass surgery with or separately from death

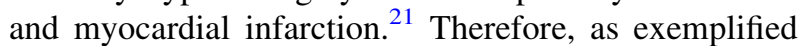

in the current report by Lassen et al., ${ }^{1}$ we owe to our patients such compulsive attention to technical and physiologic details for assessing the physiologic severity of focal and diffuse CAD to guide management and procedures. For other centers using other protocols, such reproducibility and ischemia or outcomes-driven thresholds are essential as the basis for PET quantitative perfusion and nuclear cardiology to grow into a future potentially definitive role for managing CAD.

\section{References}

1. Lassen ML, Rasmussen T, Christensen TE, Kjaer A, Hasbak P. Respiratory gating in cardiac PET: Effects of adenosine and dipyridamole. J Nucl Cardiol 2016.

2. Handel RC, et al. ACCF/ASNC/ACR/AHA/ASE/SCCT/SCMR/ SNM 2009 appropriate use criteria for cardiac radionuclide imaging: a report of the American College of Cardiology Foundation Appropriate Use Criteria Task Force, the American Society of Nuclear Cardiology, the American College of Radiology, the American Heart Association, the American Society of Echocardiography, the Society of Cardiovascular Computed Tomography, the Society for Cardiovascular Magnetic Resonance, and the Society of Nuclear Medicine. Circulation. 2009;119:e561-87.

3. De Bruyne B, Fearon WF, Pijls NH, Barbato E, Tonino P, Piroth Z, Jagic N, Mobius-Winckler S, Rioufol G, Witt N, Kala P, MacCarthy P, Engström T, Oldroyd K, Mavromatis K, Manoharan G, Verlee P, Frobert O, Curzen N, Johnson JB, Limacher A, Nüesch E, Jüni P. FAME 2 trial investigators. Fractional flow reserve-guided PCI for stable coronary artery disease. N Engl J Med. 2014;371:1208-17.

4. van Nunen LX, Zimmermann FM, Tonino PA, et al. Fractional flow reserve versus angiography for guidance of PCI in patients with multivessel coronary artery disease (FAME): 5 year follow up of a randomised controlled trial. Lancet. 2015. doi: 10.1016/S01406736(15)000574.

5. De Bruyne B, Baudhuin T, Melin JA, Pijls NH, Sys SU, Bol A, Paulus WJ, Heyndrickx GR, Wijns W. Coronary flow reserve calculated from pressure measurements in humans. Validation with positron emission tomography. Circulation. 1994;89:1013-22.

6. Loghin C, Sdringola S, Gould KL. Common artifacts in PET myocardial perfusion images due to attenuation-emission misregistration: clinical significance, causes and solutions in 1177 patients. J Nucl Med. 2004;45:1029-39.

7. Gould KL, Pan T, Loghin C, Johnson N, Guha A, Sdringola S. Frequent diagnostic errors in cardiac PET-CT due to misregistration of ct attenuation and emission PET images: a definitive analysis of causes, consequences and corrections. J Nucl Med. 2007;48:1112-21.

8. Gould KL, Pan T, Loghin C, Johnson NP, Sdringola. Reducing radiation dose in rest stress cardiac PET-CT by single post stress cine $\mathrm{CT}$ for attenuation correction-quantitative validation. J Nucl Med 2008;49:738-45.

9. Johnson NP, Pan T, Gould KL. Shifted helical CT to optimize cardiac PET-CT co-registration: quantitative improvement and limitations. J Mol Imaging. 2010;9:256-67.

10. Johnson NP, Gould KL. Partial volume correction incorporating $\mathrm{Rb}-82$ positron range for quantitative myocardial perfusion PET based on systolic-diastolic activity ratios and phantom measurements. J Nucl Cardiol. 2011;18:247-58.

11. Kitkungvan D, Vejpongsa P, Korrane KP, Sdrigola SS, Gould KL. Clinical utility of enhanced relative activity recovery on systolic 
myocardial perfusion SPECT-lessons from PET. J Nucl Med. 2015;56:1882-8.

12. Bacharach SL, Carson RE. In hotblood-quantifying the arterial input function. JACC Cardiovasc Imaging. 2013;6:569-73.

13. Vasquez AF, Johnson NP, Gould KL. Variation in quantitative myocardial perfusion due to arterial input selection. J Am Card Cardiol CV Imaging. 2013;6:559-68.

14. Johnson NP, Gould KL. Regadenoson versus dipyridamole hyperemia for cardiac positron emission tomography (PET) imaging. J Am Coll Cardiol Imaging. 2015;8:438-47.

15. deKemp RA, Yoshinaga KY, Beanlands RSB. Will 3-dimensional PET-CT enable the routine quantification of myocardial blood flow. J Nucl Cardiol 2007;14:380-97.

16. Renaud JM, DaSilva JN, Beanlands RSB, deKemp RA. Characterizing the normal range of myocardial blood flow with rubidium-82 and N-13 ammonia PET imaging. J. Nucl Cardiol. 2013;20:578-91.

17. Yoshida K, Mullani N, Gould KL. Coronary flow and flow reserve by positron emission tomography simplified for clinical application using Rb-82 or N-13 ammonia. J Nucl Med. 1996;37:1701-12.

18. Gould KL, Johnson NP, Bateman TM, Beanlands RS, Bengel FM, Bober R, Camici PG, Cerqueira MD, Chow BJW, Di Carli MF,
Dorbala S, Gewirtz H, Gropler RJ, Kaufmann PA, Knaapen P, Knuuti J, Merhige ME, Rentrop KP, Ruddy TD, Schelbert HR, Schindler TH, Schwaiger M, Sdringola S, Vitarello J, Williams KA, Gordon D, Dilsizian V, Narula J. Anatomic versus physiologic assessment of coronary artery disease: role of CFR, FFR, and PET imaging in revascularization decision-making. J Am Coll Cardiol. 2013;62:1639-53.

19. Gould KL, Johnson NP. Quantitative coronary physiology for clinical management: the imaging standard. Curr Cardiol Rep. 2016;18:1-10. doi:10.1007/s11886-015-0684-7.

20. Gould KL, Johnson NP, Kaul S, Kirkeeide RL, Mintz GS, Rentrop KP, Sdringola S, Virmani R, Narula J. Patient selection for elective revascularization to reduce myocardial infarction and mortality: new lessons from randomized trials, coronary physiology, and statistics. Circ Cardiovasc Imaging. 2015;8:e003099. doi: 10.1161/CIRCIMAGING.114.003099.

21. Taqueti VR, Hachamovitch R, Murthy VL, Naya M, Foster CR, Hainer J, Dorbala S, Blankstein R, Di Carli MF, Global coronary flow reserve associates with adverse cardiovascular events independently of luminal angiographic severity, and modifies. The effect of early revascularization. Circulation. 2015;131:19-27. 